\title{
Digital Transformation of Small and Medium Sized Enterprises Production Manufacturing
}

\author{
Manel Koumas $^{1,2}$, Paul-Eric Dossou ${ }^{2,3^{*}}$, Jean-Yves Didier ${ }^{1}$ \\ ${ }^{1}$ IBISC Laboratory, University Paris-Saclay, Evry, France \\ ${ }^{2}$ Department of Technological and Societal Transition, ICAM Paris-Sénart, Lieusaint, France \\ ${ }^{3}$ SPLOTT, AME, University Gustave Eiffel, Marne-La-Vallée, France \\ Email: *paul-eric.dossou@icam.fr
}

How to cite this paper: Koumas, M., Dossou, P.-E. and Didier, J.-Y. (2021) Digital Transformation of Small and Medium Sized Enterprises Production Manufacturing. Journal of Software Engineering and Applications, 14, 607-630.

https://doi.org/10.4236/jsea.2021.1412036

Received: November 7, 2021

Accepted: December 14, 2021

Published: December 17, 2021

Copyright $\odot 2021$ by author(s) and Scientific Research Publishing Inc. This work is licensed under the Creative Commons Attribution International License (CC BY 4.0).

http://creativecommons.org/licenses/by/4.0/ (c) (i) Open Access

\begin{abstract}
Industry 4.0 concepts have brought about a wind of renewal in the organization of companies and their production methods. However, this integration is subject to obstacles when it comes to Small and Medium sized Enterprises-SMEs: the costs of new technologies to be acquired, the level of maturity of the company regarding its level of digitization and automation, human aspects such as training employees to master new technologies, reluctance to change, etc. This article provides a new framework and presents an intelligent support system to facilitate the digital transformation of SMEs. The digitalization is realized through physical, informational, and decisional points of view. To achieve the complete transformation of the company, the framework combines the triptych of performance criteria (cost, quality, time) with the notions of sustainability (with respect to social, societal, and environmental aspects) and digitization through tools to be integrated into the company's processes. The new framework encompasses the formalisms developed in the literature on Industry 4.0 concepts, information systems and organizational methods as well as a global structure to support and assist operators in managing their operations. In the form of a web application, it will exploit reliable data obtained through information systems such as Enterprise Resources Planning-ERP, Manufacturing Execution System-MES, or Warehouse Management System-WMS and new technologies such as artificial intelligence (deep learning, multi-agent systems, expert systems), big data, Internet of things (IoT) that communicate with each other to assist operators during production processes. To illustrate and validate the concepts and developed tools, use cases of an electronic manufacturing SME have been solved with these concepts and tools, in order to succeed in this company's digital transformation. Thus, a reference model of the electronics manufacturing companies is being developed for facilitating the future digital transformation of these domain companies. The realization of these use cases and the new ref-
\end{abstract}


erence model are growing up and their future exploitation will be presented as soon as possible.

\section{Keywords}

Industry 4.0, Small and Medium Enterprises, Human-Machine Interface, Cyber-Physical System, Artificial Intelligence, Internet of Things,

Information Systems, Advanced Robotics, Lean Manufacturing, DMAIC

\section{Introduction}

Small and medium-sized enterprises (SMEs) play a major role in the growth of the economy of countries such as France. In the context of globalization and competitiveness due to emerging countries, Industry 4.0 concepts are used in large companies for increasing their performance and digitally transforming them. Thus, they could be more competitive and efficient. Despite the definition and the demonstration of the benefits to use industry 4.0 concepts for transforming companies, most SMEs have not yet decided to implement these concepts. Several obstacles to the implementation of these concepts exist such as the cost of new technologies, the academic level of employees, the fear of new technologies and their impact on employment, the lack of environmental expectations, etc. Then, it becomes essential to find the best way and responses to incite SMEs to digitally transform their production processes.

Indeed, many scientific and technological challenges must be met for the effective digital transformation of these SMEs. The digital transformation of these companies implies modernizing their facilities as well as their operating modes through the integration of new technologies. For instance, the integration of robots will allow the company to obtain more success in repetitive tasks. The use of information technology will facilitate the decision process and tasks execution. Technologies such as artificial intelligence, big data analytics, internet of things, robotics, and organizational methodologies are required for the company's digital transformation. This article proposes to optimize the production chain of these companies in terms of performance (cost, quality, lead time) by combining the technological, organizational, and scientific aspects common to all Industry 4.0 methodologies, with social, societal, and environmental dimensions (sustainable criteria) that are essential for successful implementation in SMEs. A sustainable framework and a decision aided tool containing key performance indicators have been developed for digitally transforming SMEs.

Thus, one problem to solve for the company production processes digitization is the operator's knowledge of technologies. Indeed, managers will need information for making good decisions, but operational decisions could not be executed if operators do not know how to manage their collaboration with machines, or robots. Then, it is important to solve the problem of human expectations in the integration of the company information systems and the new tech- 
nology tools that will be implemented in the manufacturing system. This article proposes the design and development of an intelligent human/machine interface that will assist the operators in their collaboration with production machines such as cobots, mobile robots, but also in their management of the company information systems. This tool is based on the artificial intelligence concepts such as deep learning, multi-agent systems and expert systems.

After a literature review focusing on the Industry 4.0 concepts, information systems, and organizational methodologies, the concepts of the sustainable Industry 4.0 that have been developed will be presented. Then, the architecture of the intelligent Human/Machine Interface allowing the integration of the company's information system and the technological tools of the production chain will be described. Then, a focus will be made on an electronic SME for solving its use case in the digital transformation and validating the concepts tools presented. The results will be enhanced as the kernel for the development of a reference model for the electronic domain companies by using case-based reasoning and generalization reasoning.

\section{Literature Review}

\subsection{Industry 4.0 Concepts}

The concept of Industry 4.0 (I4.0) is based on the integration of information and communication technologies (ICTs) and advanced industrial technologies in cyber-physical systems (CPS) to create a digital, smart, and sustainable factory [1]. Production and information systems have merged to form CPSs, which make the plant's equipment interact among themselves and with humans, and coordinate with the environment, using the Internet of Things (IoT) as a means of communication [2].

\subsubsection{Industry 4.0 Context}

The industrial world has undergone many revolutions and production has continued to evolve over time. After mechanization, mass production through electrification, and automation supported by the rise of electronics and computerization, comes the fourth industrial revolution. Known as I4.0, it aggregates concepts such as Human Machine Interaction (HMI), Artificial Intelligence (AI), advanced additive manufacturing technologies [1], Big Data and Cloud Computing [3]. Whereas Germany had a cybernetic vision of the I4.0 concept and focused on the development of CPSs, France forged it by relying on a logic of modernization of the production tool, strengthening automation in particular and betting on the integration of new technological bricks [2].

According to the implementing decree $\left(n^{\circ} 2008-1354\right)$ of article 51 of the law on the modernization of the economy, relating to the criteria for determining the category to which a company belongs for statistical and economic analysis purposes: "Small and medium-sized enterprises are those employing between 10 and 250 people and whose annual turnover does not exceed 50 million euros or whose balance sheet total does not exceed 43 million euros". They include the 
category of microenterprises which employ fewer than 10 people and whose annual turnover or balance sheet total does not exceed 2 million euros.

The introduction of I4.0 in manufacturing companies enables them to respond effectively to customer demands and requirements, and to improve their competitiveness [4]. In this context, SMEs do not know how to deal with it, and face difficulties in its implementation and struggle to take advantage of its full potential to increase their productivity [5]. To do this, SMEs need to restructure their familiar centralized decision-making mechanisms, and replace them with agile and highly adaptable manufacturing systems that offer interactive and collaborative decision-making mechanisms [6].

\subsubsection{New Technologies Integrated by Industry 4.0}

New technologies involved in industry 4.0 concepts are described as follows:

1) CPSs using self-organizing and self-controlling capabilities [7], represent computers with networks of actuators and sensors installed as embedded systems in equipment and machine parts and connected via the Internet [4] [8].

2) IoTs refers to smart connectivity of anything, anytime, anywhere. It enables interactivity between people and objects for the exchange of data and information between all objects connected to the Internet. It includes mobile connected devices to transmit real-time information about production processes such as Radio Frequency IDentification (RFID) tags, sensors, and actuators [6] [9].

3) HMI allows automation and operators to complement each other, providing technological assistance via intelligent user interfaces [10].

4) Cloud computing (CC) is used to store the large volume of data generated by CPSs and makes them accessible from anywhere. It refers to both the applications provided as services over the internet and the hardware/software systems in the data centers that provide those services [11]. It is also defined using a number of characteristics, service models and deployment models: five characteristics (on-demand self-service, broad network access, resource pooling, rapid elasticity, and measured service), four deployment models (private, community, public, and hybrid clouds) and three services models (Software, Platform, and Infrastructure As A Service "SaaS, PaaS, IaaS") [12].

5) Big Data: describes the huge amounts of data that result from the massive use of sensors and control systems in the I4.0 and their management [13].

6) Artificial Intelligence is described as "a collection of all kinds of technologies and methods, which are used to execute human brain-related tasks, especially cognitive tasks such as learning and problem-solving" [14]. It is composed of different techniques such as:

a) Expert Systems that were designed to simulate the problem-solving behavior of a human being. They are generally able to define what is expected as a solution to a problem.

b) Multi-agent systems which are composed of intelligent agents destined to solve problems. They could be used for developing simulation tools (digital twin). 
c) Machine learning which is destined to make the machine intelligent and able to learn by itself. Machine learning techniques are divided into supervised learning, unsupervised learning, and reinforcement learning.

d) Deep Learning which is a sub-part of machine learning. It is "an application of neural networks with several layers of nodes (4 or more) between input and output" [15].

7) Advanced Robotics concern Robots that use artificial intelligence and concepts such as machine learning capable of performing tasks of a high level of complexity with minimal human interaction.

8) Extended Reality uses technology to make information interactive by adding an overlay of digital content and relevant information.

9) Simulation: Virtually simulates the concepts, application and construction of prototypes and processes using computer resources.

10) Cybersecurity: It is both the insecurity created by cyberspace and the technical and non-technical practices to make it more secure [16]. Cybersecurity concerns the defensive and offensive uses of information systems that irrigate modern organizations [17].

\subsubsection{Synthesis on Industry 4.0 for SMEs}

In light of recent events, it is important to consider building resilience to address the fragilities in productive interdependencies that have been revealed by the COVID-19 health crisis since 2019 [18] [19], and SMEs can adapt under any circumstances, while remaining flexible and innovative in their manufacturing practices. The last two years have indeed been marked by one of the largest and most severe disruptions in the history of the industrial world. The COVID-19 pandemic revealed existing vulnerabilities in the production system [20]. The technological advances offered by I4.0 make it possible to effectively address some of these risks and companies can proactively manage them and continue their operations [21].

These new technologies described above are mainly deployed in big companies but need to be adapted to SMEs by finding solutions to the brakes of their managers for the industry 4.0 concepts implementation. In this article, a sustainable framework including these technologies, but also social, societal, and environmental aspects will be developed for accelerating the levers of industry 4.0 concepts implementation in SMEs.

\subsection{Information Systems}

The use of new technologies in companies involves to make them collaborating with different information systems, for increasing the level of communication between managers, and employees, thus improving the enterprise performance with more flexibility and configurability.

\subsubsection{Integration of the Company Production System}

Using IoT is all about networking the system and leveraging horizontal and ver- 
tical integrations to gain efficiencies [22]. Horizontal integration allows machines, IoT devices, and engineering processes to cooperate seamlessly by ensuring the network between individual machines, equipment, and production units. Vertical integration concedes production data to be used to make decisions about activity, personnel, and other criteria by enabling communication between the horizontally integrated network and other systems, such as ERP, MES, etc. by ensuring network beyond the classic production hierarchy levels, from the sensor level to the business level of the company.

\subsubsection{The Lean Information System}

The notion of a Lean Information System is found in the literature and characterizes it as being agile to react to fluctuations and hazards, open to ensure a partnership with suppliers, customers, and partners and interoperable to facilitate communication between the different systems and reconcile the different business facets. However, the company's information system (IS) is made up of a multiplicity of software applications and each system aims to meet a given objective for a particular business facet, and is developed according to its own business specifications, without any standardization. This leads to redundancy, heterogeneity, and an increase in the volume of information, resulting in risks of inconsistency, rigidity of the IS and great difficulty in communicating in the context of inter-company collaboration. The Industrial Internet of Things (IIoT) offers a solution to this problem. Indeed, one of its key advantages is that it can serve as the foundation for a wide range of smart manufacturing solutions. Machine learning (ML), artificial intelligence of things (AIoT), Big Data, analytics, manufacturing execution systems (MES), enterprise resource planning (ERP), digital twins and many other applications can all be built on or access an IIoT foundation. It can also leverage and extend existing technologies. Machine-to-machine communications, sensors and their data, automation, and control systems have been around for a very long time [23].

IIoT functions as a kind of middleware that enables communication between devices, machines, controllers, databases, user-oriented applications such as HMI and information systems, and people. It also enables the collection, aggregation, visualization, analysis, and transmission of large amounts of data and their context, in near-real time, to the systems and applications that need them. Herein lies the link between IIoT and information systems such as MES and ERP systems, which execute transactions, provide data, drive analytics, and support real-time visibility. With this capability, edge computing solutions become more convenient and easier to integrate with the cloud.

\subsubsection{Synthesis on the Information Systems for SMEs}

ERP is an operational system that manages production through dedicated IT applications. MES is a workshop supervision system. It includes various functionalities, in particular product traceability and genealogy [24] [25]. Production management and control systems are necessary to provide the global context for 
IIoT. The same is true for automation and control systems. All of them complement each other to provide the most complete view of what is happening in the smart factory.

As presented above many information systems exist and could be exploited for increasing the company performance. But their use in SMEs and their impact have to be developed. In addition, the lack of operators' knowledge on the use of these information systems and new technologies presented above corresponds to a problem to solve in these companies for improving their performance. In this paper, a solution to this problem will be proposed through the development of a human machine interface.

\subsection{Organizational Methodologies}

Manufacturing companies are usually inserted in a highly competitive environment, in which new challenges are constantly emerging, such as the advent of disruptive concepts and technologies [26]. In this context, manufacturers need to increase their quality and productivity levels, while reducing costs, in order to respond quickly to increasing market demands [27]. To help them achieve these goals, several approaches have been developed, such as Lean Manufacturing (LM) and, more recently, Industry 4.0 (I4.0).

Industry 4.0 offers significant technological advances that enable real-time integration between all participants in the value chain, making them more productive, intelligent, and agile [26]. It is characterized by an increased level of automation and data exchange, through the exploitation of I4.0 technologies, which allow production systems to be modular and flexible. This results in mass production of highly customized products [4] [28].

\subsubsection{Lean Manufacturing Methodology}

Lean Manufacturing (LM) has been widely used and disseminated in different industries. It aims to eliminate waste in the value chain, improve quality and productivity, and produce in accordance with customer expectations [29] [30]. It can be conceptualized as a set of practices and principles that help companies organize and control their production [31], allowing them to reduce resource use and maximize revenue, thereby generating positive impacts on stakeholders [32].

LM is one of many approaches that help manufacturers address the challenges of implementing and integrating I4.0 technologies into SMEs production. Its role as well as its importance in the implementation of Industry 4.0 has been clearly demonstrated [33] [34]. LM and I4.0 complement each other at a conceptual level and I4.0 tools support lean methods [34]. Some of the traditional barriers can be overcome, increasing the chances of successful implementation [35]. Even though the LM approach is structured to be simplistic, it could be, in addition to the integration of new technologies, more difficult for operators due to the cadence (takt time). Thus, the high level of automation owing to I4.0 concepts involves managing operators reorganization and during the methodology 
implementation, thinking about ergonomics and people quality of life in working stations. Indeed, both approaches seek to improve productivity, quality, focus on eliminating waste, and are customer-oriented [36]. LM practices (or Lean Practices-LP) are positively associated with I4.0 technologies, enabling performance improvements [28] [37]. The implementation of these principles and practices is linked to improved operational performance [38] [39] [40].

LM is an approach that encompasses a variety of manufacturing practices, aimed at reducing process variation [41], identifying and eliminating waste along the value chain [29] [31] [38] [42]. Principles are the elements of the strategic level and represent the ideals and laws of the system. In [29], the author identifies five basic principles for maximizing value and eliminating waste: specifying value from the customer's perspective; identifying the value stream; circulating value; producing to customer demand; and striving for perfection. These principles are operationalized by Lean Practices [43] that seek to reduce waste and variability in manufacturing processes, resulting in improved business performance [41].

\subsubsection{Lean Manufacturing Practices}

Many tools are integrated in lean manufacturing methodology and already show their effectiveness in the company improvement process. Some of them are Value Stream Mapping (VSM), Single Minute Exchange of Dies (SMED), 5S system, Visual Management, Kanban, Andon, etc. [44] [45] [46]. They are used to facilitate the development of better sustainable manufacturing strategies and ensure the activity of improving it [47].

Thereby, SMED represents one of the techniques of LM. It is a systematic method of analyzing and optimizing changeover times in order to improve the Synthetic Performance Time (SPT) of the equipment and to reduce the size of the batches. This method corresponds to an organization according to the logic of the flow drawn in response to the request of the customers and requires the installation of short and diversified series. Several people could work simultaneously in the same space and respect the production planning. Also known as rapid tooling change, it describes the entire process of converting a production machine from one product to another. The tooling changeover time is the time from the last good part produced in the previous run, at normal line speed, to the first good part in the next run, at maximum line speed.

\subsubsection{DMAIC Methodology}

In addition to the powerful lean approach that is primarily focused on eliminating waste (Muda), another equally powerful tool is used essentially for eliminating inconsistencies (Mura). This is Six Sigma, which contributes to the improvement of financial and operational performance as well as customer satisfaction by reducing substandard products and services [48]. The implementation of Six Sigma process improvement projects is based on the DMAIC methodology (Define, Measure, Analyze, Improve, and Control). This model provides a 
sequence of problem management steps to follow using quality tools. The project process is realized step by step [49]. The effectiveness of Six Sigma is characterized by the structural rigor and discipline of DMAIC [50]. This tool is a learning model and manages how to collect, treat, and analyze data for solving problems.

\subsubsection{Synthesis on the SME Organizational Improvement Approach}

Each method presented above has advantages that could be exploited for transforming digitally the SMEs. They could be combined for increasing their impact during the transformation. In this article a combination of lean manufacturing (and its tools) and DMAIC is proposed as an organizational solution for structuring the SMEs. The following part will focus on the exploitation of the literature review for developing concepts and methodologies in order to digitally transform SMEs.

\section{Concepts and Methodology}

This part focuses on the concepts extracted from the previous literature review that could contribute to defining an adaptive methodology of SME digital transformation. A general framework for sustainable industry 4.0 concepts implementation in these companies will be introduced. Then, the general process of a company's digital transformation will be presented. Finally, the architecture of the human/machine interface being developed will be explained.

\subsection{The Generic Framework for the Digital Transformation of SMES}

In the literature, many Industry 4.0 concepts focus on new technologies, and scientific or organizational theories, such as presented in [22] where Industry 4.0 concepts implementation in a company, with a horizontal integration through the value creation process, an engineering throughout the product life cycle and a vertical integration through the entire production system. This idea integrating technological, scientific, and organizational dimensions could be combined with sustainability (social, societal, and environmental dimensions) to create a new conceptual framework of Industry 4.0 more adapted to SMEs. The digital transformation of an SME goes through a progressive process in which people are at the center of the company's evolution. Three kinds of transformations have been defined for the SME digital evolution as shown in Figure 1. These transformations are:

1) The physical transformation through the integration of new technologies for improving the company physical processes with new technologies and methods such as lean manufacturing and DMAIC. The Production manufacturing system of the company could be improved step by step with the exploitation of organizational tools for reducing lead time, increasing the level of quality (process, system, and product) and reducing the production manufacturing costs. This transformation includes the use of new technologies that could be 


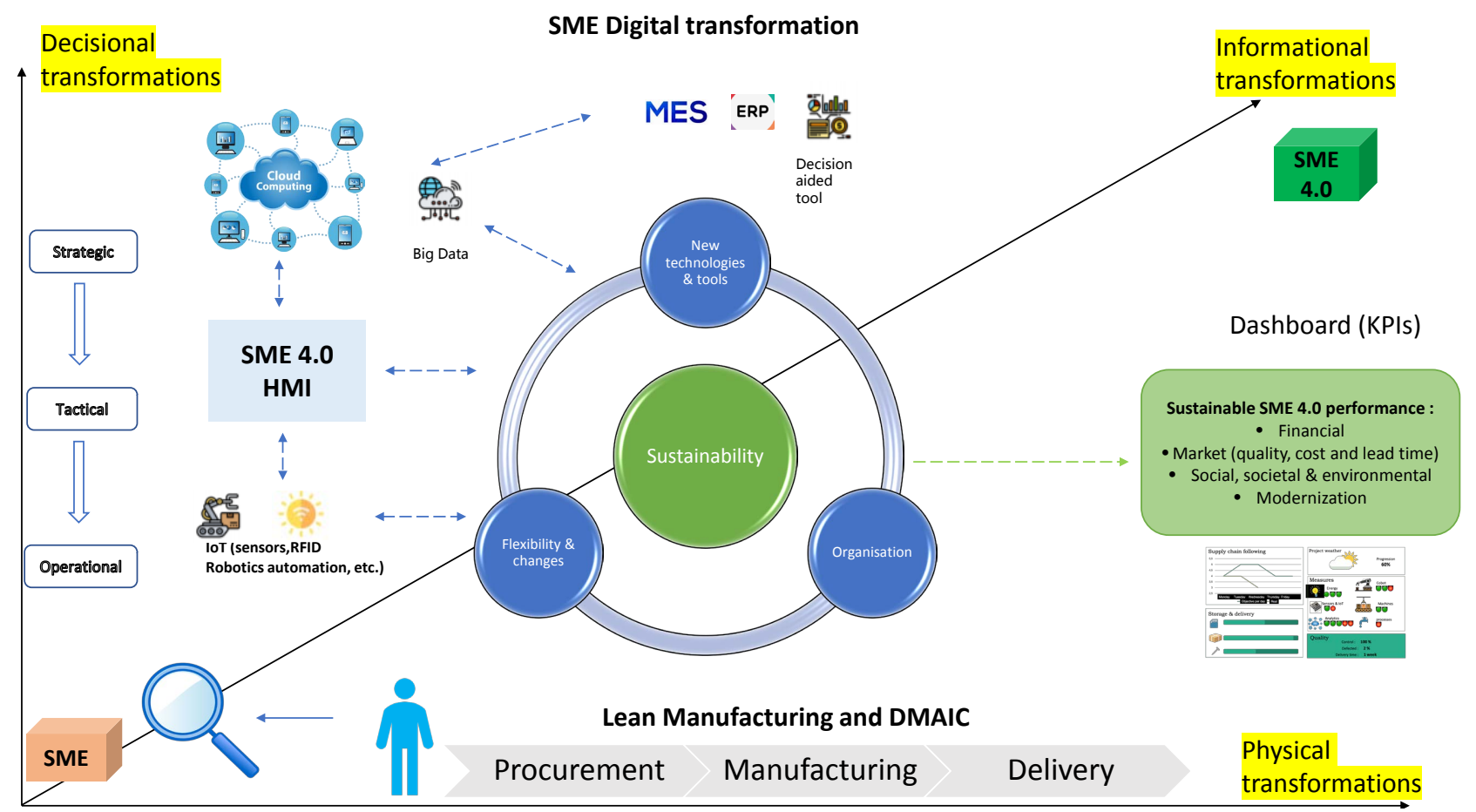

Figure 1. Generic framework for the digital transformation of SMEs.

involved in the manufacturing process such as CNC machines, robots, cobots, mobile robots, IoTs, artificial intelligence (deep learning, multi-agent systems ad expert systems). A new criterion named sustainability and composed of carbon reduction, social, societal, and environmental aspects is added in the company's physical transformation processes. This new technology integration has to be done with the objective to support operators in their tasks.

2) The decisional transformation with the structuring of strategic, tactical, and operational decisions of the company for making the company decision more consistent. Decisions in the company must be structured and linked for increasing its competitiveness. At each decision level, the company structure will be optimized for giving the best response in terms of efficiency to its transformation. Decision aided tools and intelligent systems will be developed and implemented at each level and contribute to the company performance improvement. The operators will have tools available for realizing their tasks and to take the right decisions during the production process. The managers will transform themselves into manager 4.0. They will integrate new technologies and sustainability in their management such as robotics process automation (RPA) for realizing repetitive tasks and then they could focus their attention on the management of their team.

3) The informational transformation through the integration of information system tools in the company. Information tools (MES, ERP, decision aided tool, etc.) could be chosen and implemented in the company. Indeed, the company's massive data have to be acquired with the IoTs in the production process, stored, analyzed, and treated through a data lake and a classical data warehouse for tak- 
ing convenient decisions in the company improvement process. A manager or an operator can easily ask and extract data from the data warehouse, but the data lake is required for storing and treating all non-structured data. Then, it is an advantage for taking with success real time decisions at each level. The company information system could manage data in relation with the production system and increase the efficiency of the company production process. The creation of an SME-HMI is required for integrating new technologies in the physical system thus linking the operators with machines and the information system. The objective is to facilitate the operator's work.

The use of organizational methods will also contribute to increasing the SME performance. Indeed, a hybrid methodology based on Lean manufacturing combined with DMAIC has been elaborated to digitally transform the company production manufacturing. This methodology is applied with the integration of new technologies in the production system. The performance criteria used for improving the system are based on sustainability as the kernel of the transformation and the triptych (Quality, Cost and Delivery). A scorecard with all these criteria, in addition to modernization degree criterion has been developed to measure the state of an SME at a specific moment. An example of a criterion result in the decision aided system (dashboard) is given in Figure 2. Each sector of activity, the desirable transformations to make SMEs perform in the future have been predefined and can be exploited to improve an SME.

\subsection{The Process of the SME Digital Transformation}

A digital transformation process has thus been defined with successive steps in Figure 3. The first step corresponds to the acquisition of the company's context, i.e., the recovery of all the information and data necessary for a precise understanding of its current functioning. Interviews are conducted for this purpose. The result of this phase allows us to carry out the second phase which corresponds to the modeling of the existing situation. This is the current state of the company. Then, the existing model is measured thanks to the definition of performance criteria integrating in addition to the triptych costs, quality and deadline, the social, societal, and environmental dimensions as well as the degree of modernization of the company. This measurement allows the decision aided system (dashboard) to identify dysfunctions, to highlight strengths and areas for improvement. This is the analysis stage of the existing situation. The results of the analysis phase make it possible to deduce the degree of modification of the company. This degree is directly calculated based on the results obtained from the analysis of an SME, using the criteria we have defined in the dashboard. If this degree (result of the measurement) is less than 0.25 , minimal transformations are carried out on the basis of the organizational principles of lean manufacturing, through the elimination of non-value added and the conservation of value added. If the degree of change is greater than 0.25 , then the reference model of the SME's business sector will be used for defining the progressive 


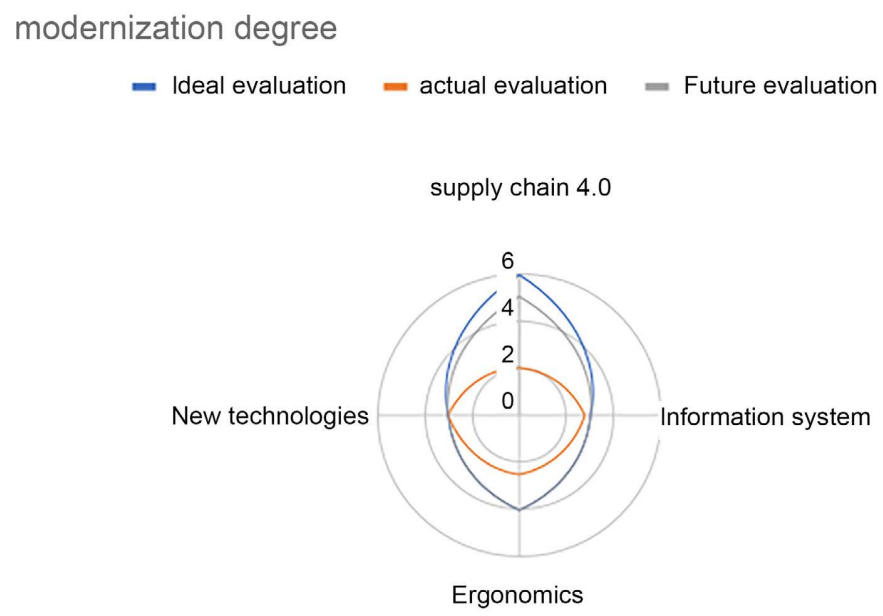

Figure 2. Example of results for modernization degree criterion.

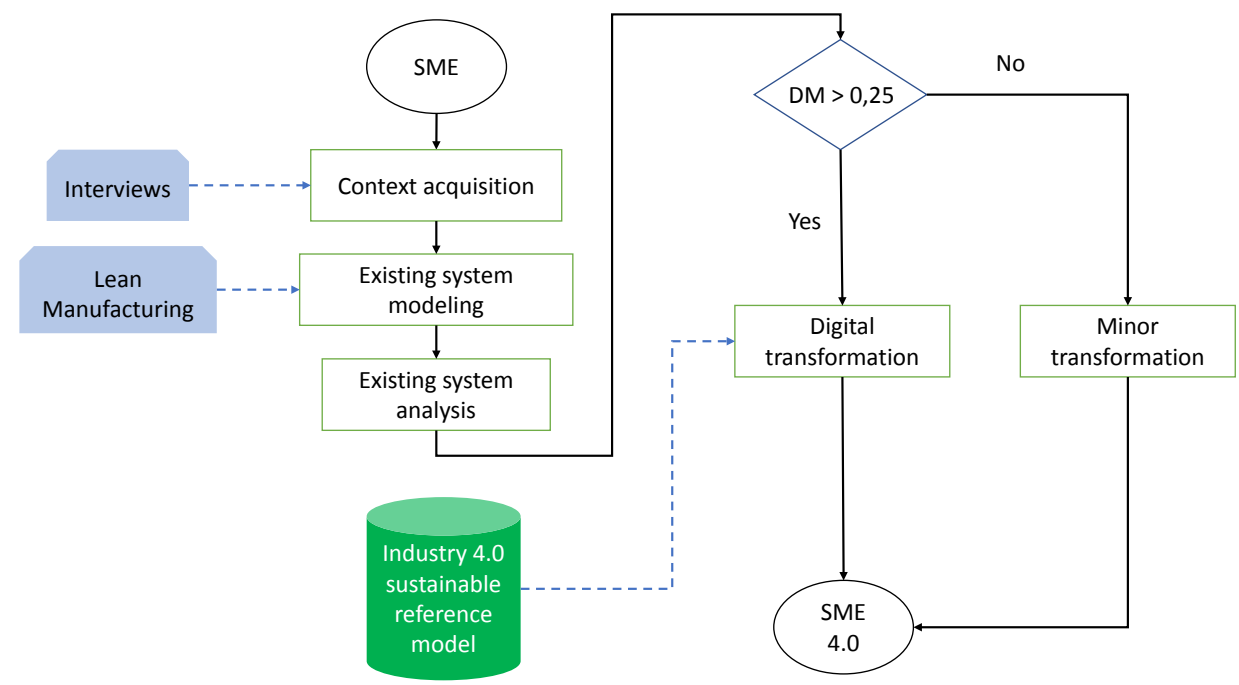

Figure 3. Sustainable and digital transformation process.

transformations that have to be implemented for achieving the SME 4.0. The design phase corresponds to the sustainable and progressive digital transformation of the company by integrating its singularities.

This design phase includes all digital transformations such as the decisional reorganization of the company with a campaign of change management and operators training, the transformation of the production system with cobots and IoT integration, the choice and the implementation of information systems, the adaptation of the human-machine interface system, the elaboration of additional specific modules, etc. The global digital transformation is made by respecting the company objectives and financial capacity, but also what is necessary for attaining the SME performance.

\subsection{The Architecture of Intelligent Human-Machine Interface System}

Improving the performance of the SME requires the transformation of internal 
and external processes of the entire supply chain, the integration of new technologies such as robots, cobots, mobile robots, the internet of things, big data analytics, etc. These new technologies aim to help people to carry out their activities, either by replacing them for repetitive or ergonomically difficult tasks, or by supporting them for those that people can easily perform. For instance, during a production phase, operators who do not have strong skills in the use and programming of robots and new technologies will find that it is difficult to directly manage the actions required for realizing production processes in collaboration with cobots. They normally had the habit of receiving their orders from the production managers (sometimes on paper). Then manufacturing orders will be sent by the information systems. The numerous information data are managed directly by the planning. In addition, all information obtained on the manufacturing system state through IoTs will be stored by using a data lake and treated for being available in a data warehouse for managers. For a better efficiency of the production system and improving the production management process, it is necessary to have an operator interface tool facilitating the execution of the tasks by the operators and the management of all their operations including the interactions with the robots and the new technologies and their reports to the hierarchy. Figure 4 represents the general structure of this interface.

Then, Industry 4.0 technologies are deployed in the SME, which allows the internal architecture to be interoperable at several levels: the equipment, the infrastructure that allows the interconnection of machines and equipment, and the reliability of information systems. A research and reflection work were carried out to determine the choice of the ERP and the MES to be used in order to fluidify the exchanges and to safeguard the important data in their context.

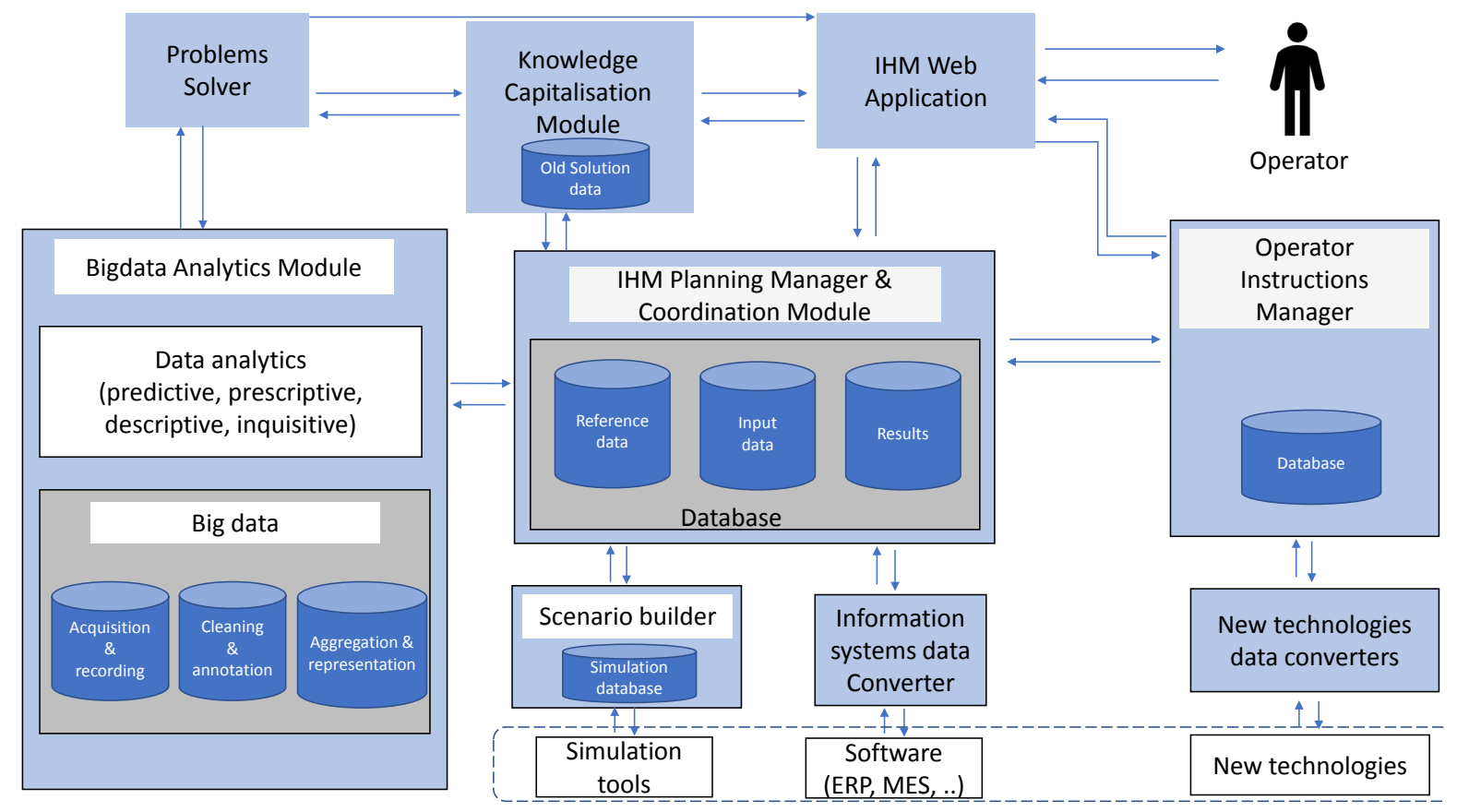

Figure 4. Architecture of the intelligent Human-Machine Interface system. 
The objective is to elaborate for operators a web application available on their smartphone, or tablet, that will give them facilities to realize their tasks and to manage their interactivity with new technology tools integrated into the production process. The SME-HMI will not change their habits but give them more flexibility and eliminate their fear of changes and their lack of experience with new technologies. This generic software as an open tool will be available for any SMEs but will require an adaptation for corresponding to the company expectations.

The intelligent SME-HMI system is composed of:

1) A visual interface which is nothing more than a web application allowing the operator to manage, without any prior knowledge in robotics or planning, the manufacturing orders, the missions of the mobile robots, the interactions with cobots and other technological tools of the production line as well as the relationship with the company's information systems such as ERP and MES. The visual interface will also show the operator the report on his performance and the state of the production system.

2) An Operator Instruction Manager-OIM module that directly links with these new technologies. This OIM can transfer information from the operator to the new technology tools such as cobots, mobile robots, or IoTs. It is the intelligent module which will transform programming into simple information or simple information to program instructions. This module is linked with the new technology data converters which really make the transformation. They both exploit technologies of deep learning for detecting expectations of the operator and comparing them to reference data related to cobots or robots programing, or IoT instructions and vice versa. The intelligent module will learn from specific situations and be able to propose new solutions.

3) An HMI manager and coordination module for coordinating and managing the relationship with information systems and simulation software tools (simulation of potential changes). The interaction between the operator and information systems is managed through this module. For instance, the manufacturing orders will be extracted from the information system and presented to the operator. The results of the production and the general state of the system will be sent to the information system and communicated to the managers. The reports and decisions will be presented to both managers and operators (decision aided tool). The information systems data converters module allows to adapt the data to the information systems and vice versa. The scenario builder module is used for simulating potential changes on the system and showing the operator the impact of his operational decision. For instance, information or orders from the operator could be integrated into the system digital twin for establishing the results expected with this decision.

4) A Big data analytics module for managing all the required data for the production and use of new technologies. Structured and unstructured data will be stored in the module, then they will be extracted, transformed, and loaded for being exploited. It contains a data lake but also a data warehouse for transferring structured data into the information system or to the operators. This module, 
after analysis, will contribute to define and suggest, to the operator, the right decisions to take and their impacts. A knowledge capitalization module is linked to this module for recording and managing all the old decisions that had been taken previously. Artificial intelligence tools such as deep learning and expert systems will be used for treating and exploiting these old cases of decisions. The problem-solving method exploited in this frame is Case-Based Reasoning. The module will propose these suggestions to the operator as an aid. A problem solver module is destined to furnish these propositions directly and uses the data from the big data analytics module as well as the data from the capitalization module to solve the operator's problems.

The concepts and methodologies elaborated and supported by the SME-HMI intelligent system are destined to consider brakes from SMEs to Industry $4.0 \mathrm{im}$ plementation and to propose to these companies' adapted methodology and tools to their digital transformation. The following part presents the application that has been made for validating concepts and improving the intelligent system being developed.

\section{Illustration}

To illustrate the concepts presented above, a focus is made on the microelectronics sector. Indeed, one SME of this activity domain has been selected for being digitally transformed. It was a win-win collaboration with the company's effective digital transformation for increasing its performance and for the research team the opportunity to validate concepts, methodology and tools elaborated in this frame. This company was a subcontractor of a leader in the airplane engine sector.

\subsection{The Company Existing System}

This company has 75 employees and turnover for 2019 (before COVID pandemic) was about 6 million euros. The number of customer orders is increasing continuously and the company for respecting these orders needs to reorganize itself. The decision to change location to a new site had been taken and the company would like at this occasion to digitally transform its manufacturing process to be more competitive. In addition, constraints and rules imposed by the customer and use cases to solve for improving the company's global performance and customer expectations are numerous. The study was based on the definition of the physical, decisional, and informational transformations necessary to make the company efficient and adapted to new technologies.

The methodology presented above and based on lean manufacturing and DMAIC was used in this study to realize the company expectations. The context acquisition was carried out based on semi-directive interviews including the operations required for the elimination of the non-added value (waste) in the company and the highlighting of the added values. The modeling stage of the existing situation allowed the whole organization of the company to be put into an analyzable format. The exploitation of performance criteria involving cost, quality, lead time, but also sustainability criteria (social, societal, and environ- 
mental) in addition to criteria measuring the degree of modernization (digital maturity) of the company could be achieved. The result of the modeling phase is summarized in the following dashboard (Table 1).

Table 1 . The company actual performance measure.

\begin{tabular}{|c|c|c|c|}
\hline \multicolumn{4}{|c|}{ Dashboard } \\
\hline SOCIAL & Ideal Evaluation & Actual Evaluation & Future Evaluation \\
\hline Career evolution & 8 & 6.5 & 7.5 \\
\hline Autonomy (decision) & 7 & 5.5 & 7 \\
\hline Quality of life at work & 9 & 8 & 9 \\
\hline CSR norm ISO 26000 & 9 & 9 & 9 \\
\hline \multicolumn{4}{|l|}{ ENVIRONNEMENT } \\
\hline Carbon footprint & 5 & 1.5 & 4 \\
\hline Recycling & 5 & 2.5 & 3 \\
\hline Environmental approach & 6 & 4 & 5 \\
\hline Respect of ISO 14001 norm & 5 & 5 & 5 \\
\hline Respect of ISO 50001 norm & 5 & 4 & 5 \\
\hline TOTAL & 26 & 17 & 22 \\
\hline \multicolumn{4}{|l|}{ QUALITY } \\
\hline Respect of ISF norm & 6 & 6 & 6 \\
\hline Validation of a product & 5 & 5 & 5 \\
\hline Validation of process & 6 & 4.5 & 6 \\
\hline Traceability & 8 & 7 & 8 \\
\hline TOTAL & 25 & 22.5 & 25 \\
\hline \multicolumn{4}{|l|}{ Cost } \\
\hline Transport cost & 4 & 0 & 0 \\
\hline Delivery cost & 6 & 0 & 0 \\
\hline Manufacturing cost & 4 & 0 & 0 \\
\hline Export cost & 4 & 0 & 0 \\
\hline TOTAL & 18 & 0 & 0 \\
\hline \multicolumn{4}{|l|}{ Lead time } \\
\hline Manufacturing time & 4 & 3 & 9 \\
\hline Storage time & 6 & 0 & 8 \\
\hline Delivery time & 4 & 0 & 7 \\
\hline Procurement time & 2 & 0 & 8 \\
\hline TOTAL & 16 & 3 & 32 \\
\hline \multicolumn{4}{|l|}{ MODERNISATION } \\
\hline Supply chain 4.0 & 6 & 1 & 5 \\
\hline Information system & 4 & 1.75 & 3 \\
\hline Ergonomics & 4 & 2.5 & 4 \\
\hline New technologies & 6 & 1 & 3 \\
\hline TOTAL & 20 & 6.25 & 15 \\
\hline
\end{tabular}




\subsection{The Analysis of the Existing System}

The analysis of the existing situation included not only the measurement of the company performance as shown in the previous table but also the detection of dysfunctions in the company, as well as the strengths and points to be improved, based on the operating rules (physical, decisional, and informational transformations). The strengths detected in the company are: the know-how of the employees, the family and artisanal dimension of the company, the good opinion of the customers, the satisfaction of the customers in terms of costs and quality. These points have to be preserved even if the global organization could be changed. The points to be improved are the reduction of delays and the elimination of non-added value (waste), the low degree of modernization of the company (no robots, no cobots, no mobile robots, old information system, no IoT system, etc.), poor decision-making structure with most decisions directly taken by the CEO, poor optimization of the company's processes. The concepts developed above have been used for transforming digitally the company such as the sustainable Industry 4.0 framework exploited for transforming the physical, decisional, and informational systems of the company and the transformation process for realizing step by step the changes in the company. The intelligent system being developed has been used for defining solutions to use cases oriented to the company modernization.

\subsection{The Digital Transformation of the Company}

As the company had a strong desire of digital transformation, some use cases were solved to help the SME in its restructuring. Thus, the use of a factory 4.0 demonstrator allowed to test and optimize the introduction of cobots on a production line of the company. For instance, a cobot has been introduced as the operator's partner for the integration of exotic components that are difficult to place on a circuit board by humans. This allows the operator to focus on easier and more value-added tasks. It has also been tested and validated the integration of a cobot in a production line for certain tasks, and its automated transfer thanks to a mobile robot to a second production line in less than 6 minutes as shown in Figure 5. The idea here is the complete automation of the cobot's movements and displacements in order to free up time for the operator. The cobots, mobile robots, sensors installed for IoT, massive data to be processed, must all remain controlled by the operator. This implies, on the one hand, the training of the latter and, as a result, an increase in salary, but also the simplification of the numerous software programs allowing this control (MobilePlanner-for the Omron mobile robot, RobotStudio-for the ABB cobot, etc.). Indeed, the operator does not have the required knowledge to program the new technology tools. It is therefore necessary to develop an interface tool to allow the operator to manage his interactions with the new technology tools.

Similarly, the operator currently receives his manufacturing orders through the company's old ERP tool. His manufacturing orders are not usable by the cobots, so unless an engineer is involved every time, the operator must be given the 


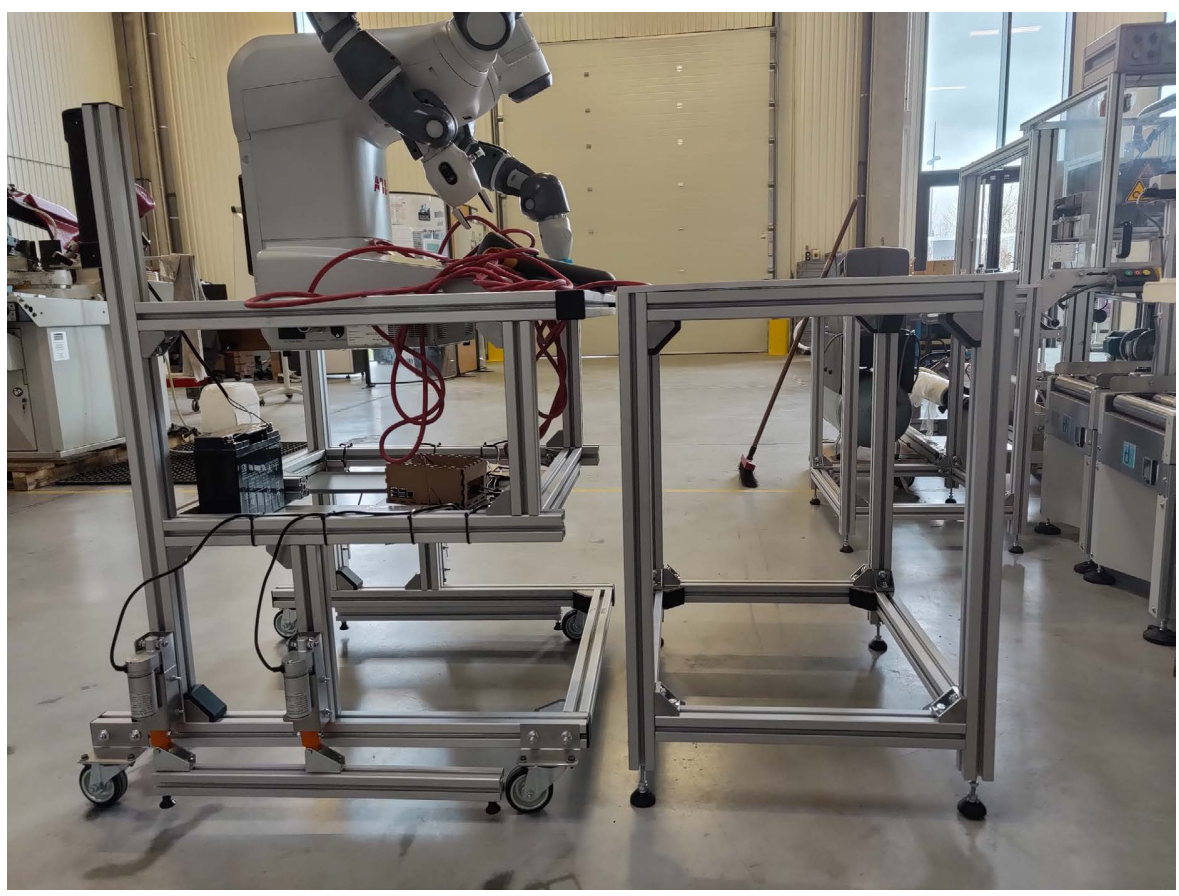

Figure 5. Development of a cobot automated transfer cart for flexibility.

possibility to easily retrieve this data and interact with the technological tools. This observation at the level of the communication between robots, operators, and information system, comforted us in the development of the intelligent system of management of the man-machine interaction presented above.

Thus, the use of intuitive user interfaces adapted to the collaboration of the operator with the machines (machine control), as well as with his exploitation of the various software is essential. Different roles (operators, supervisor/team leader, workshop manager, etc.) are considered in the design and emerging development of this ergonomic tool. Digital assistance systems should facilitate the work of operators, the communication of needs to R\&D and the communication of work measurements to supervisors.

In addition, a complete simulation of the company's production processes is being developed using FlexSim software. This simulation will represent the digital twin of the company's production system and will allow the company's operators and managers to test their suggestions before a real implementation. E.g., Figure 6 shows the packaging area for the electronic boards before they are shipped to customers.

\subsection{The Results and Expectations of the Company}

The use cases have been validated for the company. The specific virtual digital twin of the company production system shows the potential positive impact of new technologies on the company performance. The organizational improvements have already been implemented and allow to increase the SME performance. Even if the measure of the future system has not been realized, the tendency after 


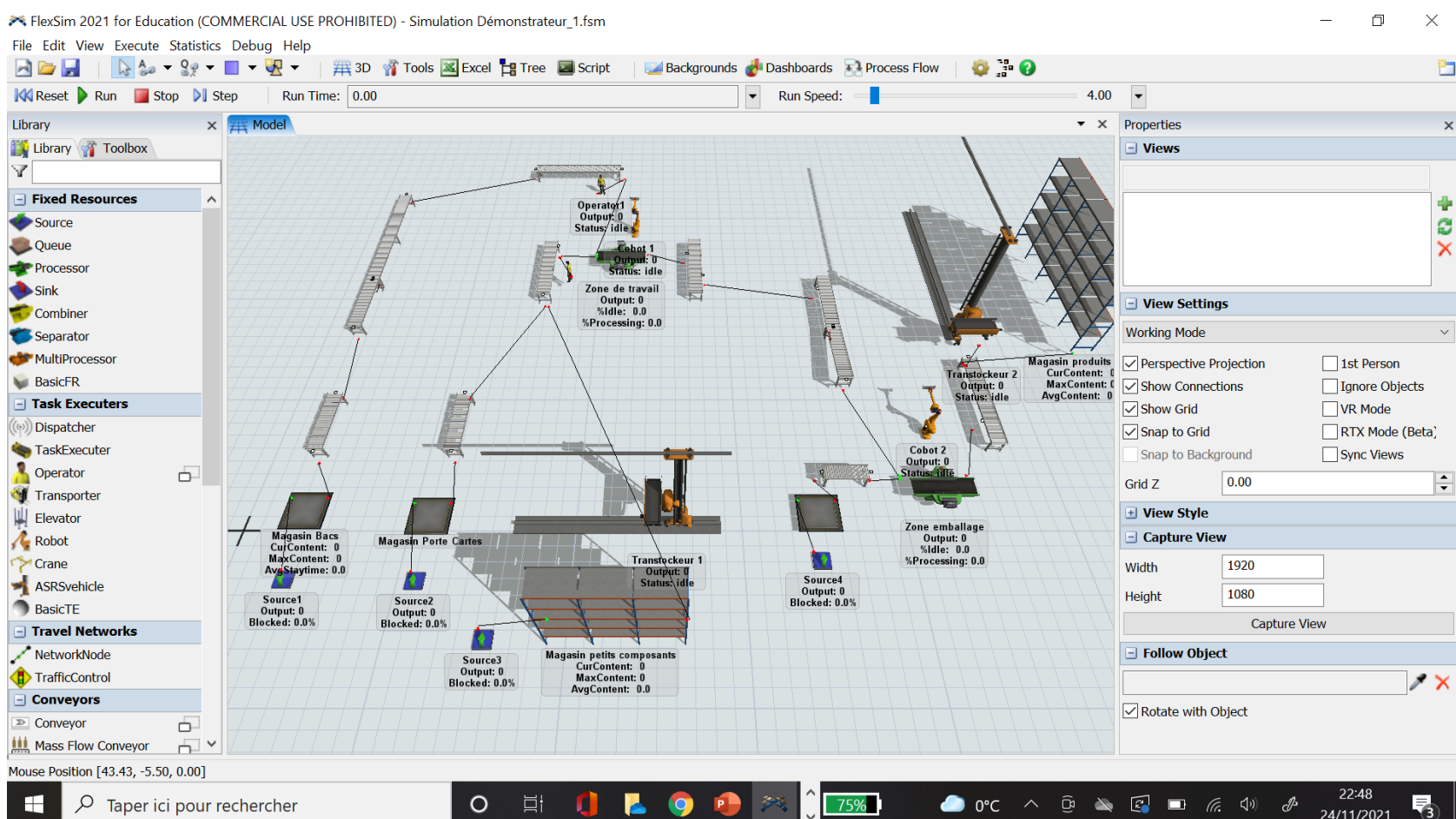

Figure 6. Virtual digital twin of packaging process.

organizational changes shows the reduction of the production cycle because of the use of Kanban, SMED and Poka-yoke (lean manufacturing tools). The use of small mobile robots allows to bring to the operators (for manual tasks) raw materials (components and cards) for their production. This organization increases the working time by reducing the operators moving. The proximity of cobots and humans at a working post has been validated through the risk analysis study that has been realized. The cobot transfer from one post to another in less than 6 minutes has been validated. The advantage is that the use of the cobot could be optimized by combining its work on two or more production lines. Then, the company global performance is increased. The company is actually preparing its manufacturing transfer to a bigger site and the implementation of all use cases solutions validated during the study.

\section{The Definition of the Reference Model for Electronics Domain Companies}

The study of this company allows the research team to obtain real data for defining a generic model for the electronics domain. The intelligent system is being developed and the reference model issued from this study has to be finished. The first version of this model has been done but as any generalization model, it needs to be improved by acquiring new knowledge. The study of another company of this sector is being realized and the results will contribute to refine the reference model and definitively validate the efficiency of the intelligent system being developed. 


\section{Conclusions}

Industry 4.0 concepts and formalisms are often used in large companies but their implementation in SMEs is in progress. Many reasons correspond to reluctance for these concepts' implementations in SMEs such as cost, human aspects, maturity of the company for new technologies implementation. In this paper, a new framework, and an intelligent support system for the digital transformation of the company have been presented. These concepts combine classical Industry 4.0 criteria (cost, lead time, quality) with sustainability as the kernel of the transformation and modernization through tools to be integrated into the company processes.

Then, for elaborating this framework, formalisms developed in the literature on Industry 4.0 concepts, information systems and organizational methods have been combined. The global structure for aiding operators in the management of their orders through data from information systems and the new technologies able to help them during the production processes has been presented. The framework and the new concepts are being implemented in an electronic manufacturing SME. Within this frame, a reference model for the electronics activity sector has been developed for being used as an aided tool to support the sustainable digital transformation of the company.

The software tool for supporting operators as a web application, for managing relationships between the information systems and new technologies, data and tools is being developed. Results of its application to the electronics company will be presented as soon as possible. Key performance indicators that have been defined for measuring the company's existing system and the future transformation have been used on the actual company organization but not yet on the future system. The future performance will be measured as soon as possible when the company will implement the new organization in its new factory.

Virtual reality tools are being developed and will be associated to this digital twin for better efficiency. The digital transformation of the company is being carried out according to the steps presented above. The final results (in the new factory) are not presented in this paper but the positive impact of new technologies introduction and the quality of the sustainable (social aspects) organization in the company let us think that the company global performance will be increased with the digital transformation according to SMEs expectations.

\section{Acknowledgements}

ICAM Paris-Sénart and IBISC Laboratory are involved in the project in collaboration with electronics sector SMEs which consider to be digitally transformed. This work was supported by grants from Région Ile-de-France (Paris-Region- $\mathrm{PhD}^{2}$ grant program).

\section{Conflicts of Interest}

The authors declare no conflicts of interest regarding the publication of this paper. 


\section{References}

[1] Zhou, K., Liu, T. and Zhou, L. (2015) Industry 4.0: Towards Future Industrial Opportunities and Challenges. 12th International Conference on Fuzzy Systems and Knowledge Discovery (FSKD), Zhangjiajie, 15-17 August 2015, 2147-2152. https://doi.org/10.1109/FSKD.2015.7382284

[2] Kohler, D. and Weisz, J.D. (2018) Transformation numérique de l'industrie: L'enjeu franco-allemand. Ifri, 145.

[3] Kagermann, H. (2014) Chancen von Industrie 4.0 nutzen. In: Bauernhansl, T., ten Hompel, M. and Vogel-Heuser, B., Eds., Industrie 4.0 in Produktion, Automatisierung und Logistik, Springer Vieweg, Wiesbaden, Vol. 4, 603-614. https://doi.org/10.1007/978-3-658-04682-8 31

[4] Kagermann, H., Wahlster, W. and Helbig, J. (2013) Securing the Future of German Manufacturing Industry: Recommendations for Implementing the Strategic Initiative INDUSTRIE 4.0. Final Report of the INDUSTRIE 4.0 Working Group, Vol. 4.

[5] Matt, D.T., Rauch, E. and Dallasega, P. (2014) Mini-Factory-A Learning Factory Concept for Students and Small and Medium Sized Enterprises. Procedia CIRP, 17, 178-183. https://doi.org/10.1016/j.procir.2014.01.057

[6] Madakam, S., Ramaswamy, R. and Tripathi, S. (2015) Internet of Things (IoT): A Literature Review. Journal of Computer and Communications, 3, 164-173. https://doi.org/10.4236/jcc.2015.35021

[7] Monostori, L. (2014) Cyber-Physical Production Systems: Roots, Expectations and R\&D Challenges. Procedia CIRP, 17, 9-13. https://doi.org/10.1016/j.procir.2014.03.115

[8] Lee, E.A. (2007) Computing Foundations and Practice for Cyber-Physical Systems: A Preliminary Report. Technical Report No. UCB/EECS-2007-72, Vol. 21, University of California, Berkeley. https://www2.eecs.berkeley.edu/Pubs/TechRpts/2007/EECS-2007-72.html

[9] Atzori, L., Iera, A. and Morabito, G. (2010) The Internet of Things: A Survey. Computer Networks, 54, 2787-2805. https://doi.org/10.1016/j.comnet.2010.05.010

[10] Gorecky, D., Schmitt, M., Loskyll, M. and Zühlke, D. (2014) Human-Machine-Interaction in the Industry 4.0 Era. 12 th IEEE International Conference on Industrial Informatics (INDIN), Porto Alegre, 27-30 July 2014, 289-294. https://doi.org/10.1109/INDIN.2014.6945523

[11] Armbrust, M., Fox, A., Griffith, R., Joseph, A.D., Katz, R., Konwinski, A., Lee, G., Patterson, D., Rabkin, A., Stoica, I. and Zaharia, M. (2010) A View of Cloud Computing. Communications of the ACM, 53, 50-58. https://doi.org/10.1145/1721654.1721672

[12] Sriram, I. and Khajeh-Hosseini, A. (2010) Research Agenda in Cloud Technologie.

[13] Babu, M.M., Rahman, M., Alam, A. and Dey, B.L. (2021) Exploring Big Data-Driven Innovation in the Manufacturing Sector: Evidence from UK Firms. Economics, Management \& Financial Markets, 16, 106-116. https://doi.org/10.1007/s10479-021-04077-1

[14] Russell, S.J., Norvig, P. and Davis, E. (2010) Uncertain Knowledge and Reasoning. In: Artificial Intelligence: A Modern Approach. 3rd Edition, Prentice Hall, Upper Saddle River, 480-692.

[15] Arel, I., Rose, D.C. and Karnowski, T.P. (2010) Deep Machine Learning-A New Frontier in Artificial Intelligence Research. IEEE Computer Intelligence Magazine, 5, 13-18. https://doi.org/10.1109/MCI.2010.938364 
[16] Cavelty, M.D. (2010) Cybersecurity. In: Burgessm, J.P., Eds., The Routledge Handbook of New Security Studies, Routledge, London, 166-174.

[17] Arpagian, N. (2015) Définition et historique de la cybersécurité. In: La cybersécurité, Presses Universitaires de France, Paris, 7-30. https://doi.org/10.3917/puf.arpag.2015.01

[18] Govindan, K., Mina, H. and Alavi, B. (2020) A Decision Support System for Demand Management in Healthcare Supply Chains Considering the Epidemic Outbreaks: A Case Study of Coronavirus Disease 2019 (COVID-19). Transportation Research. Part E, Logistics and Transportation Review, 138, Article ID: 101967. https://doi.org/10.1016/j.tre.2020.101967

[19] Pournader, M., Kach, A. and Talluri, S. (2020) A Review of the Existing and Emerging Topics in the Supply Chain Risk Management Literature. Decision Sciences, 51, 867-919. https://doi.org/10.1111/deci.12470

[20] Zhang, F., Wu, X., Tang, C.S., Feng, T. and Dai, Y. (2020) Evolution of Operations Management Research: From Managing Flows to Building Capabilities. Production and Operations Management, 29, 2219-2229. https://doi.org/10.1111/poms.13231

[21] Spieske, A. and Birkel, H. (2021) Improving Supply Chain Resilience through Industry 4.0: A Systematic Literature Review under the Impressions of the COVID-19 Pandemic. Computers \& Industrial Engineering, 158, Article ID: 107452. https://doi.org/10.1016/j.cie.2021.107452

[22] Stock, T. and Seliger, G. (2016) Opportunities of Sustainable Manufacturing in Industry 4.0. Procedia CIRP, 40, 536-541. https://doi.org/10.1016/j.procir.2016.01.129

[23] Clemons, J. (2021) The Advantages of the IIoT. Control Engineering.

[24] McClellan, M. (1997) Applying Manufacturing Execution Systems. St. Lucie Press/APICS, Boca Raton. https://doi.org/10.4324/9781498714891

[25] McClellan, M. (2000) Introduction to Manufacturing Execution Systems. MES Conference \& Exposition, Phoenix, 12-14 June 2000, 1-12.

[26] Schumacher, A., Erol, S. and Sihn, W. (2016) A Maturity Model for Assessing Industry 4.0 Readiness and Maturity of Manufacturing Enterprises. Procedia CIRP, 52, 161-166. https://doi.org/10.1016/j.procir.2016.07.040

[27] Xu, L.D., Xu, E.L. and Li, L. (2018) Industry 4.0: State of the Art and Future Trends. International Journal of Production Research, 56, 2941-2962. https://doi.org/10.1080/00207543.2018.1444806

[28] Tortorella, G.L. and Fettermann, D. (2018) Implementation of Industry 4.0 and Lean Production in Brazilian Manufacturing Companies. International Journal of Production Research, 56, 2975-2987. https://doi.org/10.1080/00207543.2017.1391420

[29] Womack, J.P., Jones, D.T. and Roos, D. (1991) The Machine That Changed the World: The Story of Lean Production. Harper Collins, New York.

[30] Jasti, N.V.K. and Kodali, R. (2015) Lean Production: Literature Review and Trends. International Journal of Production Research, 53, 867-885. https://doi.org/10.1080/00207543.2014.937508

[31] Schonberger, R.J. (2007) Japanese Production Management: An Evolution with Mixed Success. Journal of Operations Management, 25, 403-419. https://doi.org/10.1016/j.jom.2006.04.003

[32] Reuben, F., Burch, V. and Smith, B. (2019) Using Simulation to Teach Lean Methodologies and the Benefits for Millennials. Total Quality Management \& Business Excellence, 30, 320-334. https://doi.org/10.1080/14783363.2017.1303330 
[33] Sony, M. (2018) Industry 4.0 and Lean Management: A Proposed Integration Model and Research Propositions. Production \& Manufacturing Research, 6, 416-432. https://doi.org/10.1080/21693277.2018.1540949

[34] Mayr, A., Weigelt, M., Kühl, A., Grimm, S., Erll, A., Potzel, M. and Franke, J. (2018) Lean 4.0-A Conceptual Conjunction of Lean Management and Industry 4.0. Procedia CIRP, 72, 622-628. https://doi.org/10.1016/j.procir.2018.03.292

[35] Sibatrova, S. and Vishnevskiy, K. (2016) Present and Future of the Production: Integrating Lean Management into Corporate Foresight. Higher School of Economics Research Papers, No. 66/STI/2016, 22. https://doi.org/10.2139/ssrn.2854245

[36] Buer, S.V., Strandhagen, J.O. and Chan, F.T.S. (2018) The Link between Industry 4.0 and Lean Manufacturing: Mapping Current Research and Establishing a Research Agenda. International Journal of Production Research, 56, 2924-2940. https://doi.org/10.1080/00207543.2018.1442945

[37] Rossini, M., Costa, F., Staudacher, A.P. and Tortorella, G. (2019) Industry 4.0 and Lean Production: An Empirical Study. IFAC-PapersOnLine, 52, 42-47. https://doi.org/10.1016/j.ifacol.2019.11.122

[38] Liker, J.K. and Franz, J.K. (2011) The Toyota Way to Continuous Improvement; Linking Strategy and Operational Excellence to Achieve Superior Performance. McGraw-Hill, New York.

[39] Conger, S. and Miller, R. (2013) Problem-Based Learning for a Lean Six Sigma Course. All Sprouts Content, 13, 523. https://aisel.aisnet.org/sprouts all/523

[40] Kanigolla, D., A.Cudney, E., M.Corns, S. and Samaranayake, V.A. (2014) Enhancing Engineering Education Using Project-Based Learning for Lean and Six Sigma. International Journal of Lean Six Sigma, 5, 45-61. https://doi.org/10.1108/IJLSS-02-2013-0008

[41] Shah, R. and Ward, P.T. (2003) Lean Manufacturing: Context, Practice Bundles, and Performance. Journal of Operations Management, 21, 129-149. https://doi.org/10.1016/S0272-6963(02)00108-0

[42] Ohno, T. (1988) Toyota Production System-Beyond Large Scale Production. Productivity Press, New York.

[43] Bhamu, J. and Sangwan, K. (2014) Lean Manufacturing: Literature Review and Research Issues. International Journal of Operations \& Production Management, 34, 876-940. https://doi.org/10.1108/IJOPM-08-2012-0315

[44] Sanders, A., Subramanian, K., Redlich, T. and Wulfsberg, J. (2017) Industry 4.0 and Lean Management: Synergy or Contradiction? In: IFIP International Conference on Advances in Production Management Systems (APMS), Springer, Cham, 341-349. https://doi.org/10.1007/978-3-319-66926-7 39

[45] Vinodh, S., Arvind, K.R. and Somanaathan, M. (2010) Tools and Techniques for Enabling Sustainability through Lean Initiatives. Clean Technologies and Environmental Policy, 13, 469-479. https://doi.org/10.1007/s10098-010-0329-x

[46] Melton, T. (2005) The Benefits of Lean Manufacturing: What Lean Thinking Has to Offer the Process Industries. Chemical Engineering Research and Design, 83, 662-673. https://doi.org/10.1205/cherd.04351

[47] Faukner, W. and Badurdeen, F. (2014) Sustainable Value Stream Mapping (Sus-VSM): Methodology to Visualize and Assess Manufacturing Sustainability Performance. Journal of Cleaner Production, 85, 8-18. https://doi.org/10.1016/j.jclepro.2014.05.042

[48] Garza-Reyes, J.A. (2015) Lean and Green-A Systematic Review of the State of the 
Art Literature. Journal of Cleaner Production, 102, 18-29.

https://doi.org/10.1016/j.jclepro.2015.04.064

[49] Dossou, P.E., Foreste, L. and Misumi, E. (2021) Intelligent Support System for Healthcare Logistics 4.0 Optimization in the Covid Pandemic Context. Journal of Software Engineering and Applications, 14, 233-256.

https://doi.org/10.4236/jsea.2021.146014

[50] Harry, M.J., Mann, P.S., De Hodgins, O.C., Hulbert, R.L. and Lacke, C.J. (2010) Practitioner's Guide to Statistics and Lean Six Sigma for Process Improvements. Wiley-Blackwell Edition, Hoboken, 832 p. 